\title{
Os Efeitos de Lexicalidade, Freqüência e Regularidade na Leitura de Crianças Falantes do Português Brasileiro
}

\author{
The Effects of Lexicality, Frequency, and Regularity in Brazilian Portuguese Speaking \\ Children
}

\author{
Cláudia Nascimento Guaraldo Justi ${ }^{* a}$ \& Francis Ricardo dos Reis Justi ${ }^{b}$ \\ ${ }^{a}$ Universidade Federal de Pernambuco \& ${ }^{b}$ Universidade Federal de Alagoas
}

\begin{abstract}
Resumo
Os efeitos de lexicalidade, freqüência, regularidade e a interação entre esses últimos foram investigados por meio de uma tarefa de leitura administrada em crianças brasileiras que cursavam o correspondente a $3^{\mathrm{a}}$ e $4^{\mathrm{a}}$ séries do ensino fundamental. Computou-se o TR e a porcentagem de erros na leitura em voz alta de palavras e de pseudopalavras isoladas. Observou-se o efeito de freqüência e de lexicalidade, mas não o de regularidade e nem a interação entre frequiência e regularidade. Com base nesses resultados e nos resultados de outros estudos, hipotetizou-se que o efeito de regularidade, no português brasileiro, se restringe às séries iniciais. Além disso, questionou-se também a adequação de uma classificação categórica de regularidade em contraposição a uma classificação contínua.

Palavras-chave: Leitura em voz alta; Efeito de freqüência; Efeito de regularidade.
\end{abstract}

\begin{abstract}
This study investigated the effects of lexicality, frequency, regularity, and the interaction of the two latter ones on a reading aloud task conducted with Portuguese-speaking children from $3^{\text {rd }}$ and $4^{\text {th }}$ grades. The reaction time (RT) and error percentage scores on reading aloud isolated words and pseudowords were computed. The data analysis revealed the effects of frequency and lexicality on reading aloud, but not the effect of regularity nor the frequency vs. regularity interaction effect. Based on these results and on results of other studies, it was hypothesized that the regularity effect exists only at first grades for Brazilian Portuguesespeaking children. In addition, it was questioned the adequacy of a categorical classification of regularity in the light of a continuum classification.

Keywords: Reading aloud; Frequency effect; Regularity effect.
\end{abstract}

Ler é processar informação: transformar um input ortográfico em um output fonológico ou semântico. Segundo Coltheart (2005), qualquer pessoa que aprendeu a ler com sucesso possui um sistema de processamento de informação que pode executar tais transformações. Desse modo, para se compreender a leitura, é necessário entender a natureza desse sistema de processamento de informações. Os modelos computacionais têm desempenhado um papel chave no estudo científico da leitura, pois ao tentar simular o desempenho dos leitores, permitem explorar as implicações de hipóteses específicas no que diz respeito às representações e aos processos básicos envolvidos na aquisição e no desempenho da leitura (Plaut, 2005).

Dentre os diversos fenômenos documentados a respeito da leitura competente, os efeitos de lexicalidade, de freqüência e de regularidade têm recebido atenção especial dos pesquisadores que trabalham com modelagem compu-

"Endereço para correspondência: Rua Engenheiro Mário de Gusmão, 1295/104, Ponta Verde, Maceió - AL, Brasil, CEP 57035-000. Tel.: (82) 3215 1813. E-mail: claudia.ngjusti@gmail.com. Os autores desenvolveram esse trabalho como bolsistas de doutorado do Conselho Nacional de Desenvolvimento Científico e Tecnológico (CNPq). tacional. O efeito de lexicalidade refere-se a uma diferença entre a leitura de palavras e de pseudopalavras (seqüências de letras construídas com estrutura ortográfica possível na língua em questão, mas não associadas a nenhum significado), com as palavras sendo lidas com mais rapidez e precisão do que as pseudopalavras. Já o efeito de frequiência refere-se a uma vantagem, em termos de processamento, para palavras que ocorrem mais frequentemente no vocabulário escrito. Palavras que são vistas mais frequentemente (palavras de alta freqüência) tendem a ser reconhecidas mais rápida e corretamente do que aquelas que são vistas menos frequentemente (palavras de baixa freqüência). $\mathrm{O}$ efeito de regularidade refere-se ao fato de que palavras irregulares (palavras contendo uma ou mais correspondência grafema-fonema ambígua) são lidas mais lentamente e com menos precisão do que palavras regulares (palavras que obedecem às regras de correspondência grafema-fonema). Por fim, o efeito de frequiência interage com o efeito de regularidade, pois, para as palavras mais freqüentes, o efeito de regularidade é menos acentuado (Seidenberg, Waters, Barnes, \& Tanenhaus, 1984).

Há disponíveis na literatura diversos modelos de reconhecimento visual de palavras. No entanto, os modelos 
mais influentes são os modelos conexionistas de processamento paralelo distribuído - PPD (Harm \& Seidenberg, 2004; Plaut, McClelland, Seidenberg, \& Patterson, 1996; Seidenberg \& McClelland, 1989) e o modelo de dupla-rota em cascata - DRC (Coltheart, Rastle, Perry, Langdon, \& Ziegler, 2001). Considerando-se a importância dos efeitos de lexicalidade, freqüência e regularidade, a seguir são descritos, brevemente, os modelos PPD e DRC, enfatizandose suas predições quanto a esses efeitos.

O DRC é um modelo computacional que computa a pronúncia de um estímulo escrito via dois procedimentos: um procedimento lexical (conhecido como 'rota lexical') e um procedimento não lexical (conhecido como 'rota fonológica'). A rota lexical é responsável pela leitura de palavras familiares e executa essa operação acessando uma representação da palavra-alvo no léxico ortográfico. Esse processo implica ir de um conjunto de letras impressas à seleção de um único item estocado na memória lexical ortográfica. A memória lexical ortográfica é uma espécie de "dicionário mental" contendo entradas para todas as palavras que um leitor conhece (Coltheart, 2005; Coltheart et al., 2001).

Conforme mencionado, a rota lexical é responsável pela leitura de palavras familiares. Para que uma palavra desconhecida ou uma pseudopalavra seja corretamente pronunciada, um procedimento não lexical deve ser acionado. A rota fonológica do modelo DRC aplica regras de correspondência grafema-fonema ao input para converter grafemas ${ }^{1}$ em fonemas. Essa rota trabalha letra a letra, da esquerda para a direita, sendo seu funcionamento mais lento do que o da rota lexical (Coltheart et al., 2001). A rota não lexical, ou seja, a rota fonológica converte corretamente pseudopalavras e palavras regulares para sua forma fonológica. Uma palavra irregular é "regularizada" pelo procedimento não lexical - isso é, sua pronúncia será baseada em regras - o que gerará uma pronúncia incorreta da palavra.

Para explicar o efeito de freqüência na leitura em voz alta, modeladores do DRC propuseram que o acesso à entrada no léxico ortográfico para palavras de alta frequiência é mais rápido do que o acesso para palavras de baixa freqüência. Isso acontece porque palavras de alta freqüência atingem mais rapidamente o limiar de ativação especificado no modelo. Assim sendo, quanto mais freqüente for uma palavra, mais rápido será o acesso a ela (Coltheart et al., 2001).

O modelo de dupla-rota atribui a existência do efeito de regularidade na leitura em voz alta ao fato de que as duas rotas (lexical e fonológica) geram informações conflitantes quando uma palavra é irregular, mas não quando uma palavra é regular. A solução desse conflito leva tempo e é responsável pelo efeito de regularidade (Coltheart et al., 2001).

Quanto à interação entre o efeito de frequiência e o efeito de regularidade, de acordo com o DRC, palavras de baixa

${ }^{1}$ Coltheart (1978) usou o termo "grafema" para se referir a qualquer letra ou sequiência de letras que representam um único fonema $(\mathrm{CH} \rightarrow / \mathrm{x} /)$. frequiência de ocorrência apresentam um efeito de regularidade maior, pois o processamento lexical é relativamente mais lento nessas palavras e há mais tempo para a informação conflitante da rota fonológica afetar a leitura (Coltheart et al., 2001).

No que se refere ao efeito de lexicalidade, embora, no DRC, as palavras também possam ser lidas pela rota fonológica, na leitura competente, elas são lidas pela rota lexical (que é mais rápida e eficiente do que a fonológica). Já as pseudopalavras, por não terem suas representações arquivadas no léxico ortográfico, podem ser lidas apenas pelo procedimento fonológico, causando, dessa forma, o efeito de lexicalidade (Coltheart et al., 2001).

Outra classe de modelos de reconhecimento visual de palavras deriva-se da abordagem conexionista e engloba diversos modelos conhecidos como modelos de processamento paralelo distribuído (PPD). De uma forma geral, em modelos PPD de leitura, as unidades (ortográficas, fonológicas e semânticas) são ligadas umas as outras para formar uma rede. Quando um conjunto de letras é apresentado como input, as unidades correspondentes são ativadas e essa ativação se espalha para outras unidades (representando informação fonológica ou semântica). As conexões entre as unidades contêm pesos que determinam o quanto da ativação é passada adiante. As relações entre os códigos fonológico, ortográfico e semântico são probabilísticas e a aprendizagem é dirigida pela freqüência e similaridade dos padrões. Os pesos das conexões entre as unidades refletem os efeitos da exposição a muitos padrões ao invés da aprendizagem de um conjunto de regras (como é o caso no modelo DRC). Dessa forma, o conhecimento em tais redes é representado por esses pesos e aprender, de maneira bem resumida, significa ajustar os pesos com base na experiência (Plaut et al., 1996; Seidenberg \& McClelland, 1989).

Uma das principais características que distingue os modelos PPD do modelo de dupla-rota é a arquitetura que cada um emprega. De acordo com Harm e Seidenberg (2004), em termos de arquitetura, enquanto o modelo de dupla-rota (Coltheart et al., 2001) assume que há dois mecanismos separados envolvendo diferentes tipos de conhecimento e processos, a arquitetura de modelos como o de Plaut et al. (1996) e o de Seidenberg e McClelland (1989) é homogênea, no sentido de que todas as computações envolvem o mesmo tipo de estrutura (representações distribuídas de códigos ortográficos, fonológicos e semânticos) e as mesmas equações governando o espalhamento da ativação ao longo dos pesos das conexões entre as unidades. Dessa forma, em modelos PPD, um mesmo mecanismo ou processo é responsável por computar a pronúncia das palavras regulares e irregulares.

No que se refere aos efeitos de lexicalidade, de freqüência e de regularidade, bem como a interação entre esses últimos, os modelos PPD também simulam eficientemente esses efeitos. Nos modelos PPD, o efeito de freqüência acontece porque as unidades responsáveis por gerar a pronúncia de uma palavra-alvo, durante o processo de treinamento, beneficiam-se da exposição direta e repetida à própria palavra. Assim, durante o treinamento, palavras 
Justi, C. N. G. \& Justi, F. R. R. (2009). Os Efeitos de Lexicalidade, Freqüência e Regularidade na Leitura de Crianças Falantes do Português Brasileiro.

freqüentes teriam as conexões entre os seus códigos (ortográfico, fonológico e semântico) fortalecidas, com seus pesos melhor ajustados, o que resultaria em mais precisão e rapidez no processamento dessas palavras (Plaut et al., 1996; Seidenberg \& McClelland, 1989).

Assim como o efeito de freqüência, o efeito de regularidade também é devido ao fato de que as conexões que são requeridas para o desempenho correto na leitura das palavras foram ajustadas mais frequentemente na direção correta. Como as palavras são representadas por conjuntos de padrões distribuídos, durante o treinamento em uma palavra, o modelo beneficia-se não somente da exposição direta a essa palavra, mas também da exposição às palavras que compartilham padrões com essa. Como, no geral, os padrões existentes nas palavras regulares são compartilhados por um grande número de palavras da língua, espera-se que essas sejam processadas mais precisamente do que as palavras irregulares ${ }^{2}$, posto que as palavras regulares, durante o treinamento, se beneficiam tanto da exposição direta, quanto dos padrões compartilhados com outras palavras. No entanto, para palavras regulares e irregulares de alta freqüência essa diferença de processamento tende a desaparecer, pois uma alta freqüência de exposição a um item, por si só, já é capaz de proporcionar um nível "ótimo" de ajuste em seus pesos, o que faz com que o efeito de regularidade seja mais evidente para palavras de baixa freqüência (explicando, assim, a interação entre os efeitos de regularidade e de freqüência). É importante notar também que, tanto o efeito de frequiência, quanto o efeito de regularidade nos modelos PPD são derivados da mesma fonte: os efeitos de repetidos ajustes dos pesos das conexões (Plaut et al., 1996).

A explicação do efeito de lexicalidade é equivalente à explicação do efeito de freqüência. Palavras reais têm uma freqüência de ocorrência superior à das pseudopalavras (geralmente, assume-se que as pseudopalavras são equivalentes a palavras desconhecidas). Assim sendo, as palavras podem ser lidas mais rápida e precisamente do que as pseudopalavras, visto que a freqüência de exposição ao estímulo é o principal determinante dos valores dos pesos das conexões entre as unidades (Plaut et al., 1996).

Embora modelos de leitura como o de dupla-rota em cascata e o de processamento paralelo distribuído tenham sido elaborados dentro do universo da língua inglesa, algumas de suas predições são gerais o suficiente para permitir que pesquisadores os utilizem para guiar suas pesquisas em outras línguas. Nesse sentido, tornam-se importantes pesquisas realizadas no português brasileiro para se inves-

\footnotetext{
${ }^{2}$ Plaut et al. (1996), com base nos modelos PPD de leitura, argumentam a favor de uma distinção mais gradual entre palavras regulares e irregulares. De acordo com Plaut et al., podese pensar nas palavras irregulares como contendo um menor grau de consistência nas correspondências grafema-fonema, enquanto as palavras regulares guardariam um grau ótimo de consistência nessas correspondências. Dessa forma, as definições 'regular' e 'irregular' seriam vistas como dois pontos em um contínuo de consistência.
}

tigar a adequação de suas predições teóricas quanto aos efeitos de lexicalidade, frequiência e regularidade aos dados empíricos dessa língua.

Tendo como principal objetivo a investigação do desenvolvimento da leitura e da escrita em crianças brasileiras, o estudo de Pinheiro (1995) contou com uma amostra de 80 crianças da $1^{\mathrm{a}}$ a $4^{\mathrm{a}}$ série (20 crianças de cada série), julgadas por suas professoras como tendo boas habilidades de leitura e de escrita. Medidas de tempo de reação e porcentagem de erros de leitura e escrita de palavras e pseudopalavras isoladas foram a base dessa pesquisa. Tendo em vista os propósitos do presente estudo, serão relatados apenas os resultados referentes à tarefa de leitura. As palavras que compuseram a tarefa de leitura em voz alta variavam em freqüência de ocorrência, comprimento e regularidade e as pseudopalavras variavam apenas no comprimento e na regularidade. Para a análise do efeito de regularidade, Pinheiro (1995) considerou a direção da escrita, ou seja, do fonema ao grafema. É importante ressaltar que, em tarefas de leitura, a forma mais tradicional de se classificar as palavras como irregulares leva em consideração a direção grafema $\rightarrow$ fonema e não a direção fonema $\rightarrow$ grafema. Desse modo, embora o critério utilizado no estudo de Pinheiro (1995) seja adequado para tarefas de escrita, existe a possibilidade de que essa classificação dificulte a detecção do efeito de regularidade em tarefas de leitura. Para o tratamento estatístico dos dados a pesquisadora empregou uma "análise de sujeitos" e uma "análise de itens". A "análise de sujeitos" consiste em computar a média aritmética dos dados de tempo de reação dos estímulos testados, por condição experimental, para cada sujeito e fazer a análise estatística das médias resultantes. $\mathrm{Na}$ "análise de itens", computa-se a média aritmética dos dados de tempo de reação dos sujeitos, para cada item, fazendo-se a análise estatística das médias resultantes. Os tempos de reação e as médias das porcentagens de erros foram comparados por meio de uma análise de variância para medidas repetidas. A pesquisadora encontrou que o efeito de freqüência (levando-se em consideração o tempo de reação e os erros) foi significativo, em todas as séries, tanto na análise de sujeitos quanto na análise de itens. Palavras de alta frequiência foram lidas mais rápida e acuradamente do que palavras de baixa freqüência. O efeito de regularidade, considerando o tempo de reação e os erros, foi significativo apenas para as crianças da $1^{\mathrm{a}}$ e da $2^{\mathrm{a}}$ série na análise de sujeitos e na análise de itens, mas restringiu-se às palavras de baixa freqüência. Já o efeito de lexicalidade foi estatisticamente significativo para todas as séries nas comparações entre pseudopalavras e palavras de baixa freqüência, sendo as pseudopalavras lidas mais lentamente e com menos acurácia do que as palavras.

Em um trabalho subseqüente, Pinheiro (2001) classificou as crianças da $4^{\mathrm{a}}$ série do estudo de 1995 em dois grupos: leitores eficientes (13 crianças) e ineficientes (sete crianças). A partir dessa classificação, Pinheiro (2001) realizou análises individuais cujos resultados apresentaram um padrão similar ao encontrado no estudo de 1995. Isso 
é, a existência do efeito de frequiência e a ausência do efeito de regularidade para ambos os grupos (apenas duas crianças classificadas como leitores ineficientes apresentaram o efeito de regularidade).

O estudo de Capovilla, Macedo, Duduchi e Sória (1997) investigou o efeito das variáveis 'lexicalidade', 'frequiência de ocorrência', 'regularidade' e 'comprimento' em três variáveis dependentes: 'padrão de segmentação', 'tempo de reação locucional' e 'duração locucional'. Para tanto, utilizaram uma tarefa de leitura em voz alta e contaram com uma amostra de 35 estudantes brasileiros de um curso de graduação em Psicologia. O padrão de segmentação foi definido através da subtração do número de segmentos ortográficos dos itens (número de sílabas) do número de locuções produzidas pelos sujeitos durante a pronúncia desses itens. O tempo de reação locucional foi mensurado como o tempo decorrido desde a apresentação do estímulo até o início da primeira locução do sujeito e a duração locucional foi definida como o tempo decorrido desde o início da primeira locução do sujeito até o final da última locução durante a leitura de uma palavra alvo. Utilizando uma análise de variância, Capovilla et al. (1997) encontraram um efeito de lexicalidade para todas as três variáveis dependentes investigadas, isto é, as pseudopalavras produziram significativamente maior tempo de reação, maior duração locucional e maior padrão de segmentação do que as palavras. No que tange ao efeito de freqüência, as palavras de alta freqüência apresentaram um menor tempo de duração locucional do que as palavras de baixa freqüência, no entanto esse mesmo padrão não foi encontrado no tempo de reação locucional e nem no padrão de segmentação. Na variável regularidade ortográfica considerou-se, simultaneamente, tanto a direção da leitura (grafema $\rightarrow$ fonema), quanto a da escrita (fonema $\rightarrow$ grafema). Os pesquisadores encontraram que o padrão de segmentação locucional foi maior para os itens regulares do que para os itens irregulares. No que tange ao tempo de reação locucional, os itens regulares apresentaram menores médias do que os itens irregulares, no entanto essa diferença não foi encontrada no tempo de duração locucional.

O estudo de Salles e Parente (2002), realizado com 76 crianças da $2^{\mathrm{a}}$ e da $3^{\mathrm{a}}$ série, teve como objetivo analisar o uso preferencial de uma das rotas de leitura propostas pelo modelo de dupla-rota e sua possível relação com a compreensão e o tempo de leitura em diferentes grupos de leitores (bons leitores por ambas as rotas; leitores preferencialmente lexicais; leitores preferencialmente fonológicos; e maus leitores). Embora esse não fosse o foco do trabalho, as pesquisadoras reportaram os efeitos de regularidade, de freqüência e de lexicalidade na porcentagem de acertos em uma tarefa que avaliava a leitura em voz alta de palavras e de pseudopalavras isoladas. No que diz respeito à variável regularidade ortográfica, as pesquisadoras consideram a direção grafema $\rightarrow$ fonema. Levando-se em consideração a amostra como um todo e as comparações entre palavras regulares versus irregulares; freqüentes versus infreqüentes e palavras versus pseudopalavras, as pesquisadoras encontraram diferenças entre essas médias de porcentagem de acertos de $14,01 \%, 10,46 \%$ e $1,41 \%$, respectivamente. Levando em consideração apenas os dados relativos às crianças da $3^{\text {a }}$ série, a diferença encontrada entre essas médias foi de $8,18 \%, 7,08 \%$ e $5,49 \%$, respectivamente. No entanto, as pesquisadoras não apresentaram evidências quanto à significância estatística das diferenças entre essas médias, pois o estudo se concentrou em comparações entre os diferentes grupos de leitores.

O trabalho de Pinheiro, Costa e Justi (2005) teve como objetivo verificar a ocorrência do efeito de frequiência e do efeito de lexicalidade em 68 crianças da $1^{\mathrm{a}}$ à $4^{\mathrm{a}}$ série. Para tanto, os pesquisadores aplicaram uma tarefa de decisão lexical na qual o sujeito tem que decidir (pressionando uma ou outra tecla) se o estímulo que aparece no centro da tela de um computador é uma palavra ou uma pseudopalavra. Os resultados consistiram em medidas de tempo de reação para as respostas corretas e em porcentagem de erros. Para o tratamento estatístico dos dados, os pesquisadores desenvolveram uma "análise de sujeitos" e uma "análise de itens" e consideraram apenas os resultados que foram significativos em ambas as análises. Utilizando o teste $t$ para medidas repetidas, os pesquisadores encontraram que para as crianças da $1^{a}$ e da $2^{a}$ série as palavras freqüentes foram reconhecidas mais rapidamente do que as palavras infreqüentes. Já o efeito de lexicalidade específico (palavras de baixa freqüência versus pseudopalavras) foi significativo para todas as séries. Considerando a análise dos erros, o efeito de freqüência foi significativo apenas para a $2^{a}$ série e o de lexicalidade específico não foi significativo para nenhuma das séries (nesse caso, apenas a análise de sujeitos foi relatada).

Levando-se em consideração as pesquisas realizadas no português brasileiro revisadas nesse trabalho, pode-se observar que existe certa inconsistência nos resultados encontrados pelos pesquisadores, principalmente no que se refere ao efeito de regularidade. Enquanto os estudos realizados por Capovilla et al. (1997) e Salles e Parente (2002) sugerem a existência do efeito de regularidade em leitores competentes falantes do português brasileiro, incluindo crianças da $3^{\mathrm{a}}$ série (estudo de Salles \& Parente, 2002) e adultos (Capovilla et al., 1997), os estudos de Pinheiro $(1995,2001)$ sugerem que esse efeito pode estar presente apenas na leitura de crianças de $1^{\mathrm{a}}$ e $2^{\mathrm{a}}$ séries, visto que esse efeito não foi encontrado em crianças da $3^{\text {a }}$ (estudo de 1995) e $4^{a}$ séries (estudos de 1995 e 2001). No entanto, existe a possibilidade de que essa inconsistência seja mais aparente do que real, pois nos estudos de Pinheiro (1995, 2001) a direção fonema $\rightarrow$ grafema foi considerada na análise do efeito de regularidade e isso pode ter dificultado a detecção desse efeito na leitura, enquanto nos outros estudos, utilizou-se a direção grafema $\rightarrow$ fonema. Entretanto, por si só, isso não explica o porquê de Pinheiro (1995) ter detectado um efeito de regularidade na leitura de crianças de $1^{\mathrm{a}}$ e $2^{\mathrm{a}}$ séries e não em crianças de $3^{\mathrm{a}}$ e $4^{\mathrm{a}}$ séries utilizando essa mesma medida. Outro fator que dificulta a interpretação dos resultados é que na análise estatística dos dados de suas pesquisas, quase a totalidade dos pesquisadores não incluíram os itens como uma fonte de variação 
Justi, C. N. G. \& Justi, F. R. R. (2009). Os Efeitos de Lexicalidade, Freqüência e Regularidade na Leitura de Crianças Falantes do Português Brasileiro.

no termo de erro do teste estatístico utilizado, o que pode levar a um maior índice de erros do tipo I nesses estudos. Esse é um equívoco que foi observado por Clark (1973) e é conhecido na literatura como "a falácia da linguagem como um efeito fixo". Uma discussão mais pormenorizada desse erro estatístico e de como evitá-lo é apresentada na seção 'resultados' do presente estudo.

Tendo em vista essa possível inconsistência entre os resultados dos estudos realizados no Brasil quanto ao efeito de regularidade e a possibilidade de que alguns desses estudos apresentem um índice de erros do Tipo I maior do que o esperado, o presente estudo se propôs a investigar os efeitos de lexicalidade, freqüência e regularidade em crianças de $3^{\mathrm{a}}$ e $4^{\mathrm{a}}$ séries falantes do português do Brasil. Visando clarificar a situação atual, esse estudo tomou dois cuidados: na avaliação do efeito de regularidade a direção grafema $\rightarrow$ fonema foi utilizada e na análise estatística foram consideradas as observações de Clark (1973).

\section{Método}

\section{Participantes}

A amostra do presente estudo foi constituída por 49 crianças matriculadas regularmente em uma escola municipal localizada na cidade de Belo Horizonte e que se encontravam no meio do segundo ciclo (que compreende a $3^{\text {a }}$ e a $4^{\text {a }}$ série do ensino fundamental). A idade das crianças variava de 9 a 11 anos, sendo a média igual a 10 anos e 2 meses ( $D P=5,7$ meses). Dessas 49 crianças, 25 eram do sexo feminino e 24 do sexo masculino. Essas crianças foram submetidas ao subteste de leitura do TDE Teste de Desempenho Escolar (Stein, 1994) e no presente estudo considerou-se apenas os dados relativos às 26 crianças que foram classificadas como leitoras competentes, de acordo com o manual do TDE. Os dados desse estudo foram coletados como parte da pesquisa de mestrado da primeira autora (Guaraldo, 2006) que foi aprovada pelo Comitê de Ética em Pesquisa da Universidade Federal de Minas Gerais - COEP/UFMG (ETIC 375/04).

\section{Material}

Os estímulos do presente estudo (palavras e pseudopalavras) foram retirados do trabalho de Pinheiro (2003), no qual a pesquisadora, com base em sua contagem de freqüência de ocorrência de palavras (Pinheiro, 1996), lista as palavras de alta e baixa frequiência comuns no vocabulário de leitura das crianças de $1^{\mathrm{a}}$ a $4^{\mathrm{a}}$ série do ensino fundamental. As 40 palavras selecionadas para o teste de leitura de palavras isoladas são substantivos de 2 a 3 sílabas, que foram divididos de acordo com uma manipulação fatorial $2 \times 2$ da freqüência de ocorrência (alta ou baixa) e do grau de regularidade na correspondência letra/som (regular e irregular). Foram consideradas como palavras irregulares para a leitura as palavras que continham um grafema consonantal $\mathrm{X}$ e as palavras que possuíam os grafemas vocálicos $\mathrm{E}$ e O em posição paroxítona tônica (Parente, Silveira, \& Lecours, 1997). As palavras de 2 a 3 sílabas foram balanceadas entre as condições experimentais. Cada condição experimental contou com 5 palavras de 2 sílabas e 5 palavras de 3 sílabas. Tendo como base essa lista de palavras, 40 pseudopalavras que continham o mesmo número de sílabas e estrutura ortográfica semelhante à das palavras foram retiradas do trabalho de Pinheiro (2003) e compuseram o teste de leitura de pseudopalavras isoladas. Além disso, três palavras e três pseudopalavras foram utilizadas na sessão de treinamento de cada um dos testes. As palavras-estímulo utilizadas na sessão experimental encontramse em anexo (Anexo A).

\section{Procedimentos}

As crianças foram testadas individualmente, na própria escola, em uma sala cedida pela direção. O instrumento utilizado para a testagem da leitura de palavras e pseudopalavras isoladas foi um computador portátil de arquitetura compatível à IBM - PC. Um software foi elaborado para registrar o tempo de resposta (TR) e a porcentagem de erros. O TR consistiu do período de latência entre a apresentação do estímulo (palavra/pseudopalavra-alvo) e o término da resposta da criança (leitura em voz alta da palavra/pseudopalavra-alvo). Desse modo, a medida de tempo de resposta (TR) utilizada nessa pesquisa engloba as duas medidas utilizadas por Capovilla et al. (1997): o tempo de reação locucional e a duração locucional. Assim sendo, a medida de tempo de resposta empregada no presente estudo, embora não seja uma medida tradicional, é capaz de captar os efeitos de lexicalidade, frequiência e regularidade ocorram eles em quaisquer das duas medidas utilizadas por Capovilla et al. (1997). Além de registrar o TR (em milessegundos) e os erros e acertos de cada criança para cada item, o software também controlava a ordem de apresentação dos estímulos que era aleatória. As crianças eram instruídas a ler as palavras que eram apresentadas em letras minúsculas no centro da tela do computador, em voz alta, o mais rápida e corretamente possível. Imediatamente após a leitura da palavra pela criança, o experimentador apertava a tecla ' $Z$ ', se a leitura tivesse sido realizada corretamente, ou a tecla ' $X$ ', se a leitura houvesse sido feita de maneira errada ${ }^{3}$. Esse procedimento era necessário para que o programa captasse o TR e computasse os erros e os acertos das crianças em cada palavra. O mesmo experimentador aplicou os testes em todas as crianças. Cada teste durou aproximadamente 15 minutos (sessão de treinamento e experimental), sendo que a ordem de administração dos testes foi balanceada. Metade das crianças realizou, primeiro, o teste de leitura de palavras isoladas e, a outra metade, o teste de leitura de pseudopalavras isoladas.
${ }^{3}$ É importante considerar que esse procedimento pode levar a
erros por parte do pesquisador. No entanto, pode-se esperar
que esses erros se distribuam normalmente entre as condições
experimentais e desse modo, não afetem a direção dos efeitos.
Nesse caso, apenas a precisão da medida seria afetada. Por
outro lado, é importante ter em mente que outras formas de
coleta de dados não são imunes a erros. Por exemplo, não é
incomum os microfones usados nas tarefas de leitura em voz
alta falharem em captar a pronúncia dos sujeitos. 


\section{Resultados}

Existe certa discussão na literatura se na análise de dados de experimentos onde os estímulos são lingüísticos, os itens (palavras e/ou pseudopalavras) devem ser considerados um efeito fixo (Cohen, 1976) ou um efeito aleatório (Clark, 1973). De acordo com Lewis (1995), o efeito de uma variável deve ser considerado aleatório sempre que algum de seus níveis esteja sujeito a conseqüências de amostragem. Nesse sentido, no presente estudo, os efeitos das variáveis 'freqüência de ocorrência' (alta ou baixa) e 'regularidade' (regular ou irregular), devem ser considerados aleatórios, pois outras palavras (e não exclusivamente as utilizadas nesse estudo) poderiam estar representando os 2 níveis de cada uma dessas variáveis. De acordo com Clark (1973), o teste estatístico mais adequado para a análise de dados de experimentos onde os itens (palavras e/ou pseudopalavras) são considerados um efeito aleatório é o uso de $F^{\prime}$, que é uma aproximação da estatística $F$ da ANOVA. No entanto, o cálculo de $F$ ' é difícil, pois requer uma matriz de dados completa e o procedimento padrão em experimentos psicolingüísticos é excluir o TR resultante de erros. Para lidar com essa dificuldade, Clark (1973) propôs o cálculo dos limites inferior e superior de $F^{\prime}$ (min $F^{\prime}$ e max $F^{\prime}$, respectivamente). Assim sendo, sempre que min $F^{\prime}$ for significativo, $F^{\prime}$ também será e, sempre que max $F^{\prime}$ não for significativo, $F^{\prime}$ também não será. A única situação de incerteza ocorre quando min $F^{\prime}$ não é significativo, mas max $F^{\prime}$ é. Nesse caso existe a possibilidade de $F^{\prime}$ ser ou não significativo. Para se calcular min $F^{\prime}$ e max $F^{\prime}$ devese efetuar duas análises de variância: uma "análise de sujeitos" (F1) e uma "análise de itens" (F2). Obtidos os valores de $\mathrm{F} 1$ e $\mathrm{F} 2$; $\min F$ ', $\max F$ ' e seus respectivos graus de liberdade, podem ser calculados com base nesses valores conforme as fórmulas propostas por Clark (1973, p. 340). Sendo assim, no presente estudo, adotou-se como procedimento rejeitar a hipótese nula sempre que min $\mathrm{F}^{\prime}$ for significativo a um nível á igual a $5 \%$ e aceitar a hipótese nula sempre que max $F^{\prime}$ não for significativo a um nível á igual a 5\%. O programa SPSS para Windows versão 11.0 foi utilizado para se efetuar as análises de "sujeitos" e de "itens" e uma fórmula foi implementada em uma planilha do $M S$ Office Excel 2007 para os cálculos de min $F^{\prime}$ e max $F^{\prime}$.

Outro problema que se levou em consideração é que, normalmente, o TR tem uma distribuição positivamente assimétrica. Para lidar com essa questão, empregou-se o uso de medidas semi-restringidas onde todos os escores de um participante que se desviaram de sua média de TR, por mais de 2 desvios-padrão, em qualquer direção, foram limitados a esse valor (Perea, 1999). Esse procedimento alterou 3,6\% dos valores de TR desse estudo.

A média geral de TR dos participantes (incluindo palavras e pseudopalavras) foi de 1660,33 milessegundos (ms) com um desvio-padrão de 418,57 ms. A Tabela 1 apresenta a média e o desvio-padrão do TR e da porcentagem de erros dos participantes para a manipulação fatorial de freqüência e regularidade, para as palavras, para as palavras de baixa freqüência e para as pseudopalavras.

Tabela 1

Média e Desvio-Padrão do TR e da \% de Erros da Manipulação Fatorial de Freqüência e Regularidade, das Palavras, das Palavras de Baixa Freqüência e das Pseudopalavras

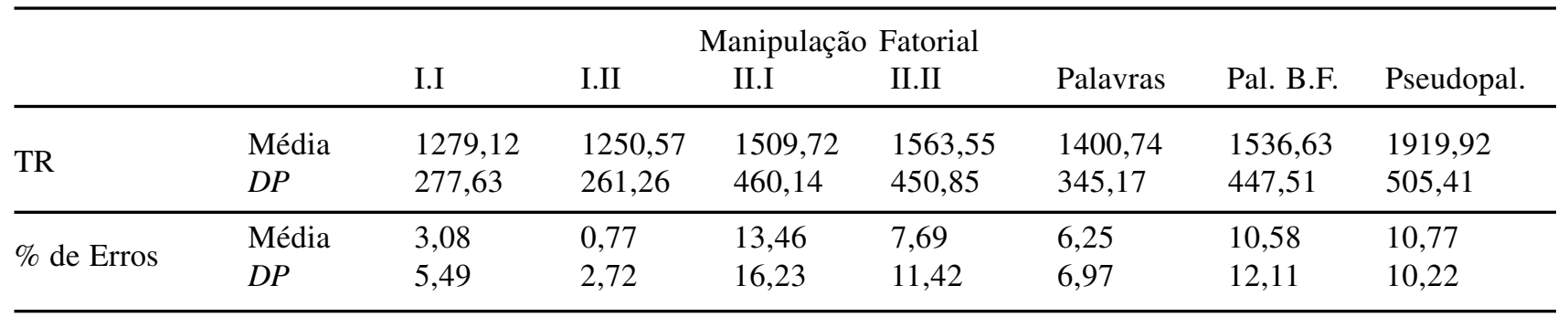

Nota. I.I: palavras de alta freqüência irregulares; I.II: palavras de alta freqüência regulares; II.I: palavras de baixa freqüência irregulares; II.II: palavras de baixa frequiência regulares.

No que tange ao efeito de freqüência, pode-se dizer que os participantes pronunciaram mais rapidamente as palavras de alta frequiência do que as palavras de baixa freqüência (ver Tabela 1). Essa diferença foi estatisticamente significativa [min $\left.F^{\prime}(1,59)=10,17 ; p<0,01\right]$. Quanto ao efeito de regularidade, não se obteve evidência estatística que apontasse uma diferença entre as médias de TR dos participantes na leitura de palavras regulares e irregulares $\left[\max F^{\prime}(23,44)=0,42 ; p>0,10\right]$. A interação entre freqüência e regularidade também não foi estatisticamente significativa $\left[\max F^{\prime}(3,42)=0,61 ; p>0,10\right]$. Considerando o efeito de lexicalidade, os participantes pronunciaram mais rapidamente as palavras de baixa freqüência do que as pseudopalavras (ver Tabela 1). A análise estatística apontou que essa diferença foi significativa [ $\min F^{\prime}(1,76)=29,7$; $p<0,001]$.

Com relação à análise de erros, a média geral da porcentagem de erros dos participantes (incluindo palavras e pseudopalavras) foi de $8,51 \%$ com um desvio-padrão de 7,60\%. Como pode ser observado na Tabela 1, pode-se dizer que os participantes pronunciaram de forma mais acurada as palavras de alta frequiência do que as palavras de baixa freqüência. Essa diferença foi estatisticamente significativa $\left[\min F^{\prime}(1,59)=5,64 ; p<0,05\right]$. Mantendo o 
Justi, C. N. G. \& Justi, F. R. R. (2009). Os Efeitos de Lexicalidade, Freqüência e Regularidade na Leitura de Crianças Falantes do Português Brasileiro.

mesmo padrão encontrado na análise do TR, o efeito de regularidade não foi estatisticamente significativo na análise da porcentagem de erros $\left[\max F^{\prime}(2,53)=2,2 ; p>0,10\right]$ e nem a interação entre freqüência e regularidade [max $F$, $(9,51)=0,92 ; p>0,10]$. Já com relação ao efeito de lexicalidade, não foi encontrada diferença estatisticamente significativa na porcentagem de erros dos participantes para a leitura de palavras de baixa frequiência em comparação à leitura de pseudopalavras $\left[\max F^{\prime}(42,82)=1,11 ; p\right.$ $>0,10]$. No entanto, ao se considerar o efeito de lexicalidade em um nível mais geral (palavras versus pseudopalavras), existe a possibilidade de que esse efeito seja estatisticamente significativo $\left[\min F^{\prime}(1,95)=2,54 ; p>0,10\right.$ e $\max F^{\prime}$ $(2,95)=3,74 ; p<0,05]$.

Como no presente estudo não se encontrou um efeito de regularidade estatisticamente significativo, decidiu-se avaliar a classificação de regularidade das palavras utilizadas nesse trabalho à luz de outro critério (Glushko, 1979; Plaut et al., 1996). Plaut et al. (1996) propõem que a distinção entre palavras regulares e irregulares seja vista como dois pontos em um contínuo de consistência. De acordo com Jared, McRae e Seidenberg (1990), a consistência de uma dada palavra depende da freqüência somada dos "amigos" dessa palavra (palavras com ortografia e pronúncia similar à da palavra-alvo) e de seus "inimigos" (palavras com ortografia similar, mas com pronúncia diferente da palavra-alvo). Com base nessa definição de consistência, avaliou-se o quão consistente eram as palavras desse trabalho que haviam sido classificadas como irregulares pelo critério de Parente et al. (1997). Para isso, foram gerados os vizinhos ortográficos ${ }^{4}$ de todas as palavras irregulares do presente estudo e adotou-se o seguinte procedimento para determinar seus índices de consistência: foram somadas a freqüência de ocorrência da palavra-alvo e dos vizinhos ortográficos que compartilhavam com ela uma pronúncia semelhante e dividiu-se esse valor pela soma total da freqüência de ocorrência da palavra-alvo e de todos os seus vizinhos. Dessa forma, o maior escore possível seria 1,00 (a palavra, nesse caso, seria o mais consistente possível, pois não teria vizinhos com pronúncia discrepante) e quanto mais próximo de zero fosse o escore da palavra-alvo, mais inconsistente ela seria. Por exemplo, considere a palavra 'cesto' que, de acordo com o trabalho de Pinheiro (1996), possui frequiência de ocorrência igual a 19. Essa palavra possui quatro vizinhos ortográficos: 'resto' (freqüência

\footnotetext{
${ }^{4}$ Nesse estudo, os vizinhos ortográficos foram gerados de acordo com o critério proposto por Coltheart, Davelaar, Jonasson e Besner (1977) no qual uma palavra vizinha é aquela que compartilha com a palavra-alvo todas as letras (preservadas as suas posições), exceto uma. Os vizinhos ortográficos das palavrasalvo desse estudo foram gerados tendo como base as palavras comuns à $3^{\mathrm{a}} \mathrm{e} 4^{\mathrm{a}}$ séries presentes no trabalho de Pinheiro (1996). Por exemplo, a palavra 'escola' tem como vizinhos ortográficos as palavras: 'esfola', 'escopa', 'esmola', 'estola', 'escora', 'escala' e 'escova'. No entanto, como de acordo com o trabalho de Pinheiro (1996) somente as duas últimas palavras são comuns à $3^{\mathrm{a}}$ e $4^{\mathrm{a}}$ série, considerou-se apenas essas palavras como vizinhas ortográficas de 'escola'.
}

204), 'cento' (freqüência 62), 'certo' (frequiência 184) e 'cesta' (freqüência 18), sendo que desses vizinhos ortográficos, apenas os mais infreqüentes, 'cento' e 'cesta', têm uma pronúncia semelhante à de 'cesto' (no que diz respeito à pronúncia do grafema vocálico E em posição paroxítona tônica). Desse modo, a palavra 'cesto' pode ser considerada bastante inconsistente, pois o seu índice de consistência é igual a 0,20 .

Com base no cálculo desse índice de consistência, foram classificadas como inconsistentes todas as palavras que apresentaram um índice de consistência menor do que um. Observou-se que das vinte palavras classificadas como irregulares nesse estudo, apenas oito apresentavam algum grau de inconsistência, sendo que dessas oito, três palavras tinham um índice próximo de 1,00 ('escola' = 0,98; 'terra' = 0,93 e 'medo' $=0,88$ ), que é o máximo de consistência possível.

\section{Discussão}

Consistente com as predições dos modelos de leitura apresentados e com os dados das pesquisas empíricas realizadas com falantes do português brasileiro pode-se observar, nesse estudo, um efeito de lexicalidade na análise do TR, onde as palavras de baixa frequiência foram lidas mais rapidamente do que as pseudopalavras. Na análise da porcentagem de erros, o efeito de lexicalidade específico não foi encontrado, existindo a possibilidade, no entanto, de que o efeito de lexicalidade em um nível mais geral seja significativo. Esse resultado talvez possa ser explicado pelo fato de que, nesse estudo, para as pseudopalavras, ao contrário das palavras, "regularizações" não foram computadas como erros (p.ex., a pseudopalavra 'sespo' poderia tanto ser lida como /såspo/ quanto como /sespo/). Assim sendo, foram menores as possibilidades de erro na leitura de pseudopalavras.

Nesse estudo, as palavras de alta freqüência foram lidas mais rápida e acuradamente do que as palavras de baixa freqüência, o que é consistente com as predições dos modelos de leitura e com os resultados de outras pesquisas realizadas com falantes do português brasileiro. A única exceção, nesse caso, é o estudo realizado por Pinheiro et al. (2005), onde não foi encontrado o efeito de frequiência para crianças da $3^{\mathrm{a}}$ e da $4^{\mathrm{a}}$ série. No entanto, o trabalho de Pinheiro et al. (2005) contou com apenas seis palavras por condição experimental, o que pode ter afetado o poder do teste estatístico na análise de itens.

Contrariando as predições dos modelos de leitura, não foi encontrado, no presente estudo, o efeito de regularida$\mathrm{de}^{5} \mathrm{e}$ nem a interação entre regularidade e freqüência. Esse 
resultado é consistente com os resultados dos estudos de Pinheiro $(1995,2001)$ onde a pesquisadora não encontrou um efeito de regularidade na leitura de crianças de $3^{\mathrm{a}}$ e $4^{\mathrm{a}}$ série. Entretanto, seria melhor comparar os resultados do presente estudo com os de Capovilla et al. (1997) e Salles e Parente (2002), pois esses pesquisadores utilizaram uma classificação de regularidade semelhante à desse estudo. Capovilla et al. (1997) relataram um efeito de regularidade no tempo de reação locucional de estudantes universitários e Salles e Parente (2002) relataram o efeito de regularidade na leitura de crianças da $3^{\mathrm{a}}$ série. No entanto, como Capovilla et al. ponderaram, seu estudo foi desenvolvido com universitários, mas utilizou uma lista de palavras adequada para a $4^{\mathrm{a}}$ série (a lista de Pinheiro, 1994) o que pode colocar em dúvida a confiabilidade de seus resultados. Além disso, enquanto Capovilla et al. (1997) realizaram apenas a "análise de sujeitos", Salles e Parente (2002), no que diz respeito às comparações entre palavras regulares e irregulares, não apresentaram evidência estatística para os resultados encontrados em seu trabalho. É importante considerar, nesse caso, que a "análise de sujeitos", como demonstrado pelas simulações Monte Carlo de Forster e Dickinson (1976) e Wickens e Keppel (1983), pode apresentar um índice de erros do tipo I mais de quatro vezes maior do que o valor nominal esperado, aumentando assim, a possibilidade de um resultado falso-positivo. Já a análise empregada no presente estudo, que envolve o cálculo de min $F$, visa exatamente se resguardar do viés positivo encontrado na análise de sujeitos e apresenta um índice de erros do tipo I próximo do valor nominal esperado (conforme os estudos de Forster \& Dickinson, 1976; Wickens \& Keppel, 1983).

Embora as considerações expostas acima façam sentido, é necessário que algumas explicações alternativas sejam consideradas. Existe a possibilidade de que o efeito de regularidade não desapareça em séries mais avançadas, mas seja apenas reduzido. Desse modo, seria possível argumentar que nos estudos de Pinheiro $(1995,2001)$ o efeito de regularidade não foi detectado na $3^{\mathrm{a}}$ e $4^{\mathrm{a}}$ séries porque a

trou muita evidência a favor dessa hipótese nos dados desse estudo, uma vez que a palavra 'exemplo' (única palavra com grafema consonantal irregular dentre os estímulos do estudo) foi lida mais rápida e acuradamente até mesmo do que a palavra 'estado' que tem o mesmo número de sílabas, também é de alta freqüência de ocorrência e é regular em sua correspondência grafema-fonema. É importante se considerar também que, com exceção do estudo de Pinheiro (1995), os outros estudos realizados com falantes do português brasileiro não colocaram a lista de palavras utilizadas em anexo, o que torna difícil avaliar essa colocação do revisor. Além disso, tendo em vista os modelos de reconhecimento visual de palavras discutidos nesse trabalho, não existiriam razões teóricas para essa diferença de processamento. O DRC (Coltheart et al., 2001) não prevê que as palavras com grafemas consonantais irregulares sejam mais difíceis de serem processadas e os modelos PPD (Plaut et al., 1996; Seidenberg \& McClelland, 1989) prevêem que é o grau de consistência das palavras que afeta o processamento e não a presença de um tipo de grafema específico. Nesse sentido, mesmo que o revisor esteja correto, os modelos estariam em dificuldades. classificação de regularidade adotada não foi a mais adequada (considerou-se a direção fonema $\rightarrow$ grafema). Já com relação ao presente estudo, o argumento seria o de que o fato do pesquisador classificar os erros on-line poderia tornar o registro dos dados menos preciso e prejudicar a detecção de efeitos de menor magnitude. Nesse caso, uma comparação com outro estudo pode ajudar a analisar as questões de precisão e poder estatístico. Dos estudos revisados o mais diretamente comparável ao presente estudo é o de Salles e Parente (2002), pois ambos utilizaram o mesmo critério de regularidade e crianças de um nível de escolaridade próximo. Em seu estudo, Salles e Parente (2002) relataram que o efeito de regularidade foi mais forte do que o efeito de freqüência. Tendo isso em mente, torna-se difícil argumentar que o presente estudo deixou de detectar o efeito de regularidade por falta de poder estatístico, uma vez que foi capaz de detectar um efeito de freqüência. Ademais, comparado aos outros estudos realizados com falantes do português brasileiro, o presente trabalho foi o único que relatou ter controlado a classe gramatical dos estímulos utilizados, o que pode ter sido uma variável confundidora nos outros estudos.

Ainda no que diz respeito ao efeito de regularidade, cabem algumas considerações a respeito do critério utilizado para classificar as palavras como regulares e irregulares. Nesse estudo, adotou-se um critério formal (categórico), tal como proposto por Parente et al. (1997). De uma forma geral, os outros trabalhos discutidos também adotaram um critério categórico semelhante a esse. No entanto, outros critérios podem ser adotados para classificar palavras como regulares ou exceções (Glushko, 1979; Plaut et al., 1996, por exemplo). O critério de Plaut et al. (1996) é inspirado em seu modelo PPD de leitura e nesse a distinção entre palavras regulares e irregulares é vista como dois pontos em um contínuo de consistência. Tendo isso em mente, procurou-se averiguar se as palavras classificadas como irregulares nessa pesquisa também seriam inconsistentes. Pôde-se observar que menos da metade dessas palavras poderiam ser classificadas como inconsistentes. Nesse sentido, se a proposta de Plaut et al. (1996) for válida e a regularidade dos estímulos for melhor capturada por um critério mais gradual que enfatize a consistência desses, não é surpreendente que o efeito de regularidade não tenha sido observado nesse estudo, posto que menos da metade das palavras irregulares desse, poderiam ser classificadas também como inconsistentes. É interessante notar que essa proposta de Plaut et al. (1996) também poderia explicar as diferenças encontradas entre as pesquisas revisadas, no que diz respeito ao efeito de regularidade. Afinal, como a consistência das palavras não foi controlada, podem existir grandes diferenças entre os estudos no que diz respeito ao índice de consistência das palavras utilizadas. Portanto, seria interessante que pesquisas futuras, além de considerarem uma distinção categórica do tipo "regular" versus "irregular", considerassem também o índice de consistência das palavras. 
Justi, C. N. G. \& Justi, F. R. R. (2009). Os Efeitos de Lexicalidade, Freqüência e Regularidade na Leitura de Crianças Falantes do Português Brasileiro.

\section{Conclusão}

De uma forma geral, no que tange as predições dos modelos de leitura quanto aos efeitos de freqüência e lexicalidade, os dados desse estudo não só corroboram essas predições como são consistentes com pesquisas anteriores realizadas com falantes do português brasileiro. No entanto, no que diz respeito ao efeito de regularidade e a interação entre regularidade e freqüência, não se encontrou nesse trabalho o padrão predito pelos modelos. Pelo menos duas hipóteses podem ser levantadas para explicar esse fato. A primeira pode ser retirada do trabalho de Pinheiro (1995) e propõe que, no caso do português brasileiro, esse efeito deve ser encontrado apenas nas séries iniciais, onde as crianças ainda não seriam leitores muito competentes e basear-se-iam principalmente na decodificação fonológica para a leitura. Assim sendo, o efeito de regularidade não deveria ser encontrado nos escores de leitura das crianças desse estudo, uma vez que podem ser consideradas leitoras competentes (eram crianças de $3^{\mathrm{a}} \mathrm{e}$ $4^{\mathrm{a}}$ série que não apresentaram dificuldade de leitura de acordo com o manual do TDE). A segunda hipótese seria a de que a distinção categórica entre regular e irregular talvez não represente a melhor distinção possível entre palavras regulares e exceção. Pode ser que a distinção entre esses dois grupos de palavras possa ser mais bem capturada por uma classificação gradual das palavras no contínuo entre dois pontos: consistente e inconsistente. Estudos futuros podem avaliar esses dois tipos de classificação e lançar luz a essa questão. Por fim, uma das limitações desse estudo é que a variável tempo de resposta mensurava processos relacionados ao acesso lexical e à produção da pronúncia dos estímulos. Desse modo, seria interessante que outros estudos investigassem se os efeitos de lexicalidade, freqüência e regularidade afetam algum desses processos em específico.

\section{Referências}

Capovilla, F., Macedo, E., Duduchi, M., \& Sória, R. (1997). Análise computadorizada de leitura em voz alta via rotas fonológica e lexical. Ciência Cognitiva: Teoria, Pesquisa e Aplicação, 1, 81-140.

Clark, H. (1973). The language-as-fixed-effect fallacy: A critique of language statistics in psychological research. Journal of Verbal Learning and Verbal Behavior, 12, 335-359.

Cohen, J. (1976). Random means random. Journal of Verbal Learning and Verbal Behavior, 15, 261-262.

Coltheart, M. (1978). Lexical access in simple reading tasks. In G. Underwood (Ed.), Strategies of information processing (pp. 151-216). London: Academic Press.

Coltheart, M. (2005). Modeling reading: The dual-route approach. In M. Snowling \& C. Hume (Eds.), The science of reading: A handbook (pp. 6-23). Malden, MA: Blackwell.

Coltheart, M., Davelaar, E., Jonasson, J., \& Besner, D. (1977). Access to the internal lexicon. In S. Dornic (Ed.), Attention and performance (Vol. 5, pp. 535-555). Hillsdale, NJ: Erlbaum.
Coltheart, M., Rastle, K., Perry, C., Langdon, R., \& Ziegler, J. (2001). DRC: A dual route cascaded model of visual word recognition and reading aloud. Psychological Review, 108, 204-256

Forster, K., \& Dickinson, R. (1976). More on the language-asfixed-effect fallacy: Monte Carlo estimates of error rates for F1, F2, F', and min F'. Journal of Verbal Learning and Verbal Behavior, 15, 135-142.

Glushko, R. (1979). The organization and activation of orthographic knowledge in reading aloud. Journal of Experimental Psychology: Human Perception and Performance, 5, 674-691.

Guaraldo, C. N. (2006). A contribuição da consciência fonológica e dos processos subjacentes à velocidade de nomeação para o desenvolvimento da leitura e da escrita no português brasileiro. Dissertação de Mestrado não-publicada, Universidade Federal de Minas Gerais, Belo Horizonte, MG.

Harm, M., \& Seidenberg, M. (2004). Computing the meaning of words in reading: Cooperative division of labor between visual and phonological processes. Psychological Review, 111, $662-720$

Jared, D., McRae, K., \& Seidenberg, M. (1990). The basis of consistency effects in word naming. Journal of Memory and Language, 29, 687-715.

Lewis, D. (1995). Análise de variância. São Paulo, SP: Harbra.

Parente, M., Silveira, A., \& Lecours, A. (1997). As palavras do português escrito. In A. Lecours \& M. Parente (Eds.), Dislexia: Implicações do sistema de escrita do português (pp. 41-55). Porto Alegre, RS: Artes Médicas.

Perea, M. (1999). Tiempos de reacción y psicología cogntiva: dos procedimientos para evitar el sesgo debido al tamaño muestral. Psicológica, 20, 13-21.

Pinheiro, A. (1994). Leitura e escrita: Uma abordagem cognitiva. Campinas, SP: Editorial Psy II.

Pinheiro, A. (1995). Reading and spelling development in Brazilian Portuguese. Reading and Writing, 7, 111-138.

Pinheiro, A. (1996). Contagem de freqüência de ocorrência e análise psicolinguísticas de palavras expostas a crianças na faixa pré-escolar e séries iniciais do $1^{\circ}$ grau. São Paulo, SP: Associação Brasileira de Dislexia.

Pinheiro, A. (2001). Heterogeneidade entre leitores julgados competentes pelas professoras. Psicologia: Reflexão e Crítica, 14, 537-551.

Pinheiro, A. (2003). Vocabulário comum de alta, média e baixa freqüência de ocorrência para crianças da $1^{a}$ à $4^{a}$ série do ensino fundamental (COCHS/DPH 168/03). Brasília, DF: Ministério da Ciência e Tecnologia

Pinheiro, A., Costa, A., \& Justi, F. (2005). Reconhecimento de palavras reais e de não-palavras em crianças de $1^{\mathrm{a}}$ a $4^{\mathrm{a}}$ série: Uma tarefa de decisão lexical. Revista de Estudos da Linguagem, 13, 145-169.

Plaut, D. (2005). Connectionist approaches to reading. In M. Snowling \& C. Hume (Eds.), The science of reading: A handbook (pp. 24-38). Malden, MA: Blackwell.

Plaut, D., McClelland, J., Seidenberg, M., \& Patterson, K. (1996). Understanding normal and impaired word reading: Computational principles in quasi-regular domains. Psychological Review, 103, 56-115.

Salles, J., \& Parente, M. (2002). Processos cognitivos na leitura de palavras em crianças: Relações com compreensão e tempo de leitura. Psicologia: Reflexão e Crítica, 15, 321-331. 
Seidenberg, M., \& McClelland, J. (1989). A distributed, developmental model of word recognition and naming. Psychological Review, 96, 523-568.

Seidenberg, M., Waters, G., Barnes, M., \& Tanenhaus, M. (1984). When does irregular spelling or pronunciation influence word recognition? Journal of Verbal Learning \& Verbal Behavior, 23, 383-404.

Stein, L. (1994). TDE: Teste de desempenho escolar: Manual para aplicação e interpretação. São Paulo, SP: Casa do Psicólogo.

Wickens, T., \& Keppel, G. (1983). On the choice of design and of test statistics in the analysis of experiments with sampled materials. Journal of Verbal Learning \& Verbal Behavior, 22, 296-309.

\section{Anexo A}

Palavras utilizadas na tarefa de leitura em voz alta

Palavras de alta freqüência de ocorrência irregulares: cabeça - escola - metro - caderno - medo - letra - terra zero - exemplo - colegas.

Palavras de alta freqüência de ocorrência regulares:

estado - caminho - coração - vida - azul - carro - frase grupo - figuras - coluna.

Palavras de baixa freqüência de ocorrência irregulares:

globo - novelo - leque - bloco - caneta - ampola - soro sarjeta - cesto - coberta.

Palavras de baixa freqüência de ocorrência regulares: gengiva - grade - nabo - tarde - brasa - caqui - caçada vasilha - bicadas - buzina. 